\title{
XII.
}

\section{Zu der platonischen Atlantissage.}

\author{
Von \\ Otto Kern in Berlin.
}

Six hat in der fünfzehnten der seiner Dissertation De Gorgone Amstelodami 1885 angehängten Thesen die Behauptung aufgestellt, dass der platonische Atlantismythos ,mutatis mutandis' auf die Niederlage der Perser zu beziehen sei. Er hat sich also der von Susemihl vor mehr als dreissig Jahren vorgetragenen Vermutung angeschlossen. Es mag sein, dass er neue Argumente beizubringen weiss, dass er sichere Beziehungen auf die Perserkriege gefunden hat, das aber steht fest, dass Susemihls und seines Schülers Brieger Gründe nicht genügen ${ }^{1}$ ). Und mag uns auch dieses und jenes an die gewaltige That des themistokleischen Athens erinnern, mag Plato unter dem Eindruck geschrieben haben, den auf ihn die Heldenthat seiner Väter gemacht hat, die Anregung zu seiner Atlantisepisode haben ihm die Perserkriege ganz gewiss nicht gegeben. Denn Plato erzählt ron einem Kriege, der vor 9000 Jahren (Timaios p. $23 \mathrm{E})^{2}$ ) zwischen den Athenern und Atlantinern geführt sei. Schon Solon hat ron dem Kriege keine directe Ueberlieferung mehr erhalten, durch ägyptische Priester hat er erst von ihm erfahren. Durch diese Zeitangabe wird die von Susemihl aufgestellte Hypothese widerlegt, nicht minder aber auch dadurch, dass das Reich der

1) Zuerst in der Uebersetzung des Kritias Stuttgart 1857, dann Genetische Entwickelung der platonischen Philosophie II 2 (1860) 4 S6 f. II $4.77 \mathrm{f}$.

3) Vgl. Susemihl Genetische Entwickelung der platonischen Philosophite 
Allantis in Westen liegt. Nach eiuem Kriege muissen wir suchen, welchen dic Athener mit westlichen Nachbarn in hohem Altertum gefïlurt haben.

Betrachten wir don Feind, mit welcheun Altathen kämpft. In Anfing des Timaios wird uns der Krieg und seine Folgen geschildert, im Kritias giebt uns Plato eine genaue Schilderung des Atlantisreiches, welches Poseidou bei der Theilung der Erde als scinen Antheil erhalten hat. Ueber die im fernen Westen wohnenden Atlantiner herrschen zehn Könige, welche Poseidon mit Kleito, der Tochter des Euenor und der Leukippe, gezeugt hat. 1)ie Etynologic des letztgenannten Frauennamens hat besondere Bedeutung: wir befinden uns im Poseidonreiche. $\mathrm{Zu}$ beachten ist auch, class dor eine Poseidonsohn Elasippos (Kritias p. $114 \mathrm{C}$ ) heisst. Auf der Insel stand ein grosser, prächtiger Tempel des Poseidon, in welchem sich ein plastisches Bild des Meergottes befand, um den hundert Nereiden auf den Rücken von Delphinen schwammen. Bei dieser Beschreibung kommen uns sofort die schönen Verse in den Sinn, in denen Ilias $\mathrm{N} 27 \mathrm{f}$. der Ritt des Poseidon durch das Neer geschildert wird, wir erinnern uns der zahlreichen Kunstwerke, auf denen wir die Bewohner des Meers in den anmutigsten Gruppierungen dargestellt sehen. Die genaue Beschreibung Platos macht es sehr wahrscheinlich, dass er ein bestimmtes Kunstwerk vor Augen hat.

Die Herrschaft der zehn Könige richtet sich (Kritias p. 119 C)

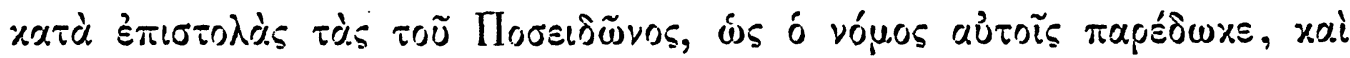

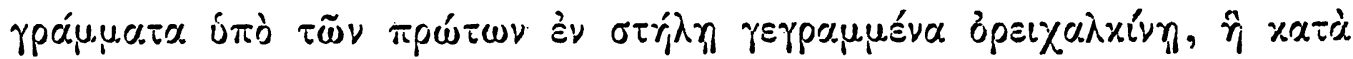

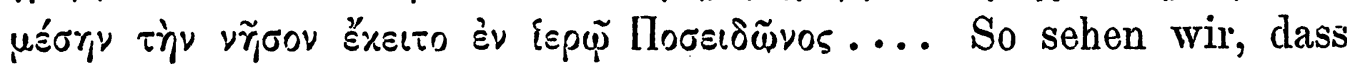
die Atlantis dem Poseidon gehört, auf ihn als seinen Stammherrn geht das Herrschergeschlecht zurück. Dass aber Athena auch im Kritias Athens Schutzgöttin ist, versteht sich von selbst,

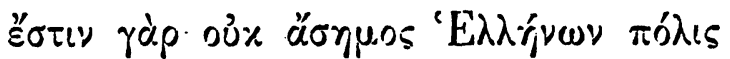

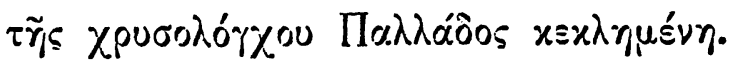

Auf der einen Seite stehen also die Poseidonsöhne, auf der anderen die Schützlinge der Athena; zwischen Beiden kommt es zu einem heftigen Kampf. In welche Zeit derselbe füllt, hat Plato selber klar angegeben, sagt er doch, dass der Kampf vor Theseus' 


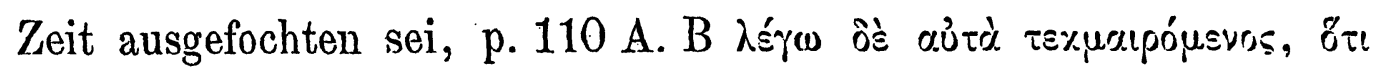

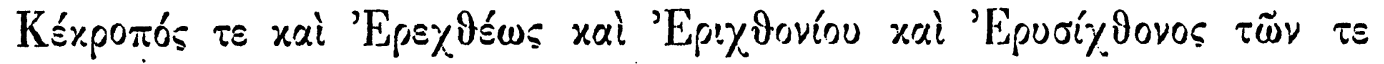

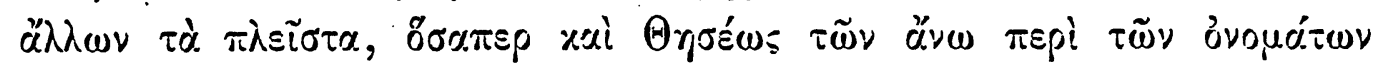

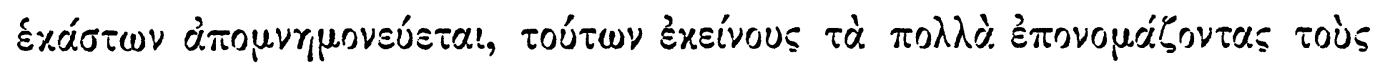

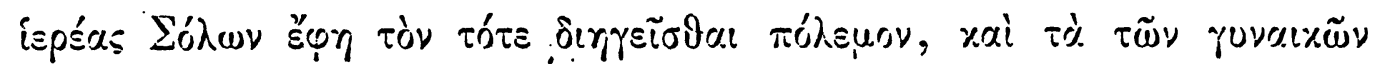

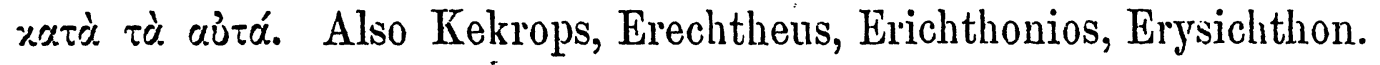
Nit Kekrops beginnen die Könige der Athener, der letzte König aus dem Geschlecht des Erichthonios ist Erechtheus. Seine Gestalt macht in der sagenhaften, ältesten Geschichte Athens Epoche, er

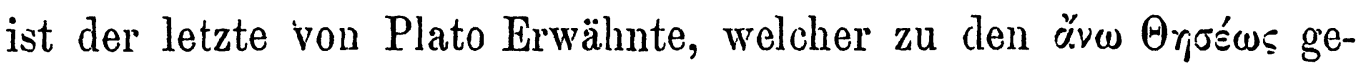
hört. Ist es verwegen, wenn wir hiernach den Krieg in die Regierungszeit des Erechtheus fallen lassen? Diese Spur zu. verfolgen wird nicht fruchtlos sein.

Vor den Perserkriegen ist kein Krieg, welchen Athen geführt hat, zu solcher Berühmtheit gelangt wie der eleusinische. Plato (Menexenos p. $239 \mathrm{~B}^{3}{ }^{3}$ ) stellt selbst beide Kriege einander gegenüber, vgl. B. Giseke Thrak. pelasgische Stämme und ihre Wanderungen S. 43. Und wenn also der Gedanke, dass der Perserkrieg in der Atlantiserzählung gemeint sei, nicht aufrecht gehalten werden kann, liegt es von Anfang an am nächsten an den eleusinischen Krieg zu denken. Der platonischen Dichtung liegt die écıs der Athena und des Poseidon zu Grunde, nach meiner Ansicht auch der Sage vom eleusinischen Krieg. So hat Isokrates Panathen. 193 auch die Sache

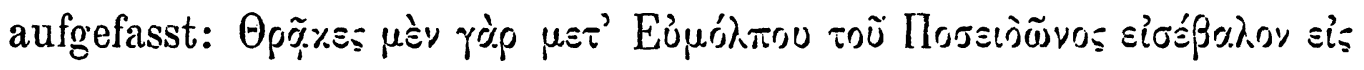

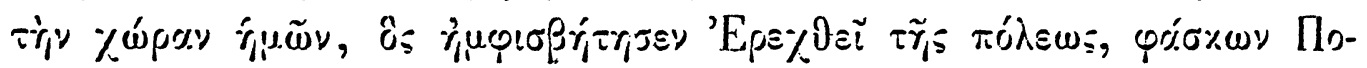

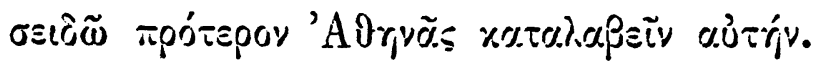

Im Atlantisreiche gebieten zehn Könige, welche die Vertreter der Perserhypothese zu Satrapen zu machen kein Bedenken getragen haben. In Eleusis herrschen vor der Vereinigung mit Athen verschiedene Fürsten, von welchen uns der homerische Demeterhymnos Triptolemos, Diokles, Eumolpos, Keleos, Polyxenos, Dolichos nennt ${ }^{4}$ ).

3) Nach den Ausführungen von Diels Abhandlungen der Preuss. Aliademie der Wissenschaften 1886 S. $21 \mathrm{ff}$. halte ich die Echtheit des Menexenos für erwicsen, anders auch jetıt Zeller Archiv I 614.

4) Vgl. Friedrich Ililler ron Gacrtringen De Graecorum fabulis ad Thraces pertineutibus Berolini 1886 p. $13 \mathrm{~s}$. 
Poscilon wurde in Eleusis hochverchrt, nach Pausanias I 38, 6 gab es dort einen Tempel des Poseidon Ilaxip. Zum Kampfe mit den Athenern hinaus ziehen die Poseidonsöhne Eumolpos und Phorbas (Harpokration u. d. Wr. (Moppáiviz!ov). Den Gegensatz zwischen den unter dem Schutz, des Poseidon kämpfenden Eleusiniern und den Athenern driicken am schärfsten aus die Worte der Praxithea in Euripideischen Erechtheus (fr. 362, 45 Nauck):

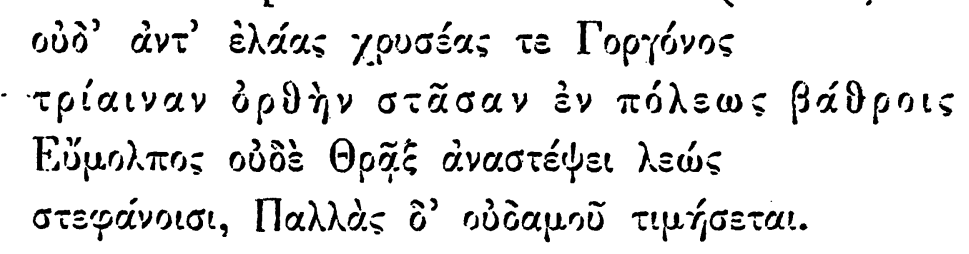

Nach diesen Erwägungen scheint es mir nicht zu kühn zu sein, wemn ich die platonische Atlantis als ein Zeugniss für den eleusinischen Krieg in Anspruch nehme. Plato hat frei mit seinem Stoff geschaltet hier, wie anderwärts; aber woher ihm die Anregung kam, wollen wir doch nicht verkennen.

Auch im Einzelnen finclen wir im Kritias Eleusis wieder. Von Demeter freilich kein Wort, ihrer scheint auch nicht im Euripideischen Stück gedacht worden zu sein, das nur den Conflict zwischen den Poseidonsöhnen und den Athenern behandelte. Aber die Fruchtbarkeit des Atlantislandes wird ganz besonders gerühmt p. $113 \mathrm{C}, 117 \mathrm{~A}-\mathrm{C}$. Sehr entschieden betont Plato den Kult der Atlantiner, und zwei gottesdienstliche Einrichtungen erinnern direct

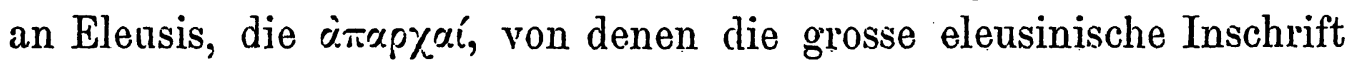
(vgl. H. Sauppe Attica et Eleusinia Ind. schol. hiem. Gottingae 1881) genaue Kunde gegeben hat, und die Jagd auf den Opferstier, welche Artemidor I 8 (vgl. A. Nebe De mysteriorum Eleusiniorum tempore et administratione publica. Diss. Halenses VIII (1886) p. 110) berichtet.

Der eleusinische Krieg ist, wie U. von Wilamowitz Aus Kydathen S. 125 sagt, 'der Kampf zweier stammfremder Völker und zweier Religionen, des thrakischen Speers wider die attische Gorgo'. So haben ihn die attischen Redner immer aufgefasst. Dasselbe gilt von dem Krieg der Atlantiner mit den Athenern, auch diese beiden Völker sind einander stammfremd, hat doch die Poseidonstatue des Atlantinertempels ein eĩos ßapßapixóv, s. Kritias p. $116 \mathrm{D}$, 
Susemihl genetische Entwickelung II 485. Die Invasion der Poseidonsöhne ist im Kritias sowohl wie in der attischen Sage ein Einfall des vereinigten westlichen Continents, wie Grote (Geschichte Griechenlands I 163, 32 Deutsche Uebersetzung) den eleusinischen Krieg mit Recht bezeichnet hat, man vergleiche nur Kritias

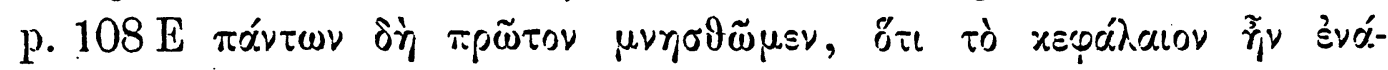

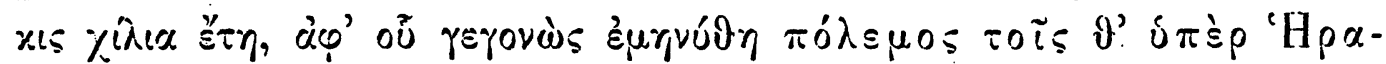

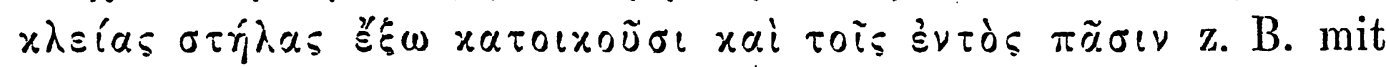

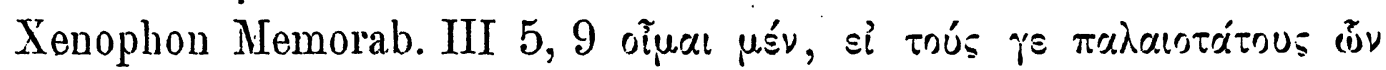

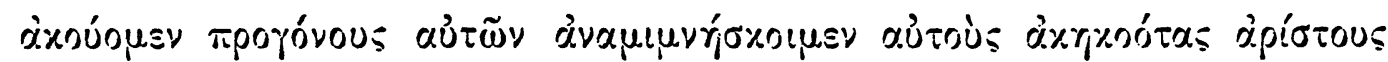

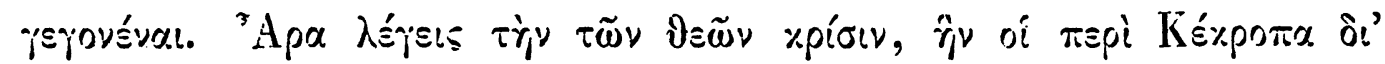

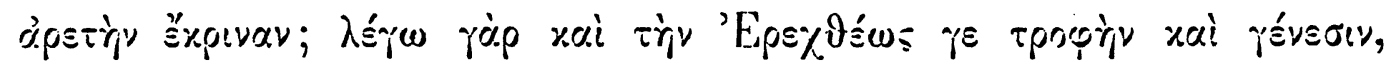

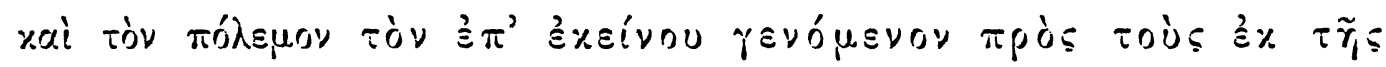

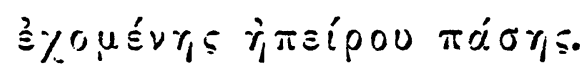

Das hohe Lob, welches v. Wilamowitz Homerische Untersuchungen S. 398 dem platonischen Kritias ertheilt hat, verdient er voll und ganz. Die Zeit, in der man dieses Meisterwerk, das ein Torso bleiben sollte, Plato absprach, ist lïngst vorüber. Mit staunender Bewunderung sehen wir, wie sein Genius aus der Anregung, welche ihm eine attische Sage gegeben, ein Werk schuf, dessen Nichtrollendung für jedes poetisch empfindende Gemüt ein unersetzlicher Terlust ist. 